\title{
Psique, soma, institución. Una lectura de la psicopatología del trabajo
}

\section{Psyche, soma, institution. A lecture on Labour Psychopathology}

\author{
Cristóbal Durán Rojas ${ }^{1}$
}

\begin{abstract}
Resumen:
En el presente artículo se exponen y discuten algunas consideraciones sobre las relaciones entre el trabajo y ciertos aspectos psíquicos, presentes en la obra del psicoanalista francés Christophe Dejours. Para trazar dicho recorrido se enfatizan aquellas expresiones sintomáticas de esta relación que estarían haciendo visible un sufrimiento experimentado por el sujeto al verse enfrentado a la tarea de su puesto de trabajo. Allí, se considera de manera central el complejo vínculo que se establece entre la institución del trabajo y las exigencias que, por su parte, impone el propio psiquismo.
\end{abstract}

Palabras claves: sufrimiento psíquico, institución, afecciones psicosomáticas.

\begin{abstract}
:
The article expose and discusses some considerations on the relationships between the labour and certain psychic aspects, present in the works of French psychoanalyst Christophe Dejours. The symptomatic expressions of this relationship are emphasized that would be making visible a suffering experienced by the subject in the task of its work position. In a central way it is considered the complex bond that settles down between the institution of labour and the demands that, on the other hand, it imposes the own psyche.
\end{abstract}

Keywords: psychic suffering, institution, psychosomatical affections.

1 Psicólogo, Universidad Diego Portales. cristobald@esfera.cl. Profesor auxiliar de Investigación Social, Universidad de Viña del mar. 


\section{Introducción.}

Existen varias razones para considerar absolutamente imprescindible llevar a cabo una reflexión en torno a los aspectos psíquicos involucrados en la actividad laboral. Pese a que ha sido un tema presente en todo el recorrido de las tradiciones que comprende la llamada Psicología de las Organizaciones, ello no impide volver a hacer preguntas en dicho ámbito, lo cual conllevaría nuevos alcances y reposicionamientos conceptuales múltiples.

La cuestión general que aquí se propone dice relación con el intercambio conceptual que se puede efectuar entre dos instancias de trabajo. Bajo el supuesto de que las enfermedades laborales son tales por tener su origen y sentido en el medio laboral, la propuesta que aquí se ensaya refiere a buscar en el psicoanálisis, como mirada conceptual, una serie de herramientas para leer e interpretar los fenómenos presentes en las situaciones laborales. En este tránsito es legítimo interrogarse si existe alguna adecuación entre la mirada del psicoanálisis y aquello que es visible por la Psicología del trabajo.

Si la Psicología del trabajo se encarga también de toda una serie de (psico)patologías que en los distintos trabajos se hacen siempre notables, por qué no intentar considerar estas patologías -estrés laboral, afecciones psicosomáticas, ansiedad, etc.- con la óptica que ya se ha desarrollado en el terreno de la observación y la investigación conceptual del psicoanálisis, para desde allí intentar responder de otro modo lo que se hace cotidiano para la sicopatología del trabajo.

Una de las razones para intentar este vínculo entre ámbitos de trabajo dice relación con aquello que en un primer momento podría ser llamado un sufrimiento del psiquismo experimentado en el vínculo con el trabajo cotidiano de un sujeto. Es allí donde algo falla, donde se muestra una dolencia que interrumpe la continuidad de la actividad laboral como actividad humana de sustento, tanto psíquico como social y económico, que aparece el sufrimiento como una modalidad de relación -entre la actividad laboral y el psiquismo- que nos hace aparecer interrogantes sobre la amplitud de este vínculo y nuestras posibilidades de intentar responder a aquellas.

Uno de los terrenos que sin duda abre enorme cantidad de preguntas y que por lo demás requiere de urgentes e ineludibles tentativas de respuesta es el ámbito de la psicopatología del trabajo. La relevancia de este gesto señalado de una "vuelta a preguntar" yace en una necesidad de intentar extender el terreno al que hacen referencias las investigaciones de la Psicología laboral y reconducirlo hacia un campo de mayor alcance y que lo compromete, a decir, la relación entre las instituciones y los aspectos psíquicos que en ellas se ven involucradas. Pero esto último requiere de una explicación adicional.

Sin el ánimo de intentar aclarar todo lo propuesto desde la entrada, se podrá decir que la institución es en un sentido amplio una actividad normada bajo ciertos patrones más o menos fijos y parcialmente estables. La actividad laboral que un sujeto desempeña en su puesto de trabajo es así, por excelencia, una institución. Sometida a horarios, a distintos encuentros y obligaciones, $\mathrm{o}$ a una gama precisa de "comportamientos esperados y esperables", esta institución se involucra absolutamente con aspectos psíquicos. Así, en toda tarea sea del tipo que sea, hay una necesaria participación del psiquismo.

Causa, efecto, producción o condición de un registro sobre el otro, de la institución sobre el psiquismo y viceversa, pueden ser maneras de enfrentar el problema, pero se puede mostrar como esto se volverá secundario al dar cuenta de la emergencia de ciertas modalidades de relación entre lo psíquico y lo laboral que se presentarían, por ejem- 
plo a través de fenómenos psicosomáticos, en los cuales se traba un compromiso excepcional entre ambas dimensiones, cristalizándose el modo de funcionar la articulación psique-soma en una actividad claramente institucionalizada.

La idea es volver, por medio de discusiones con otros alcances, a confrontar el hecho empírico que constituye los problemas subjetivos en situación laboral, sea ésta de la índole que sea, esto es, no delimitada únicamente a la empresa o a la asistencia, como terrenos más investigados por la Psicología convocada a los medios laborales. La cuestión sería no detenerse en mostrar la "anormalidad" que se puede ver en cierto vínculo como el referido, sino ante todo otorgarle visibilidad a aquello que él nos da para pensar de los límites de la relación entre el psiquismo y la institución. En este caso, y ello será importante, la institución del trabajo.

Dentro del transcurso de lo que se pretende exponer y discutir, tratar de detenerse en un autor podría parecer nada más que una excusa para hablar de ciertos problemas aparentemente ya trazados. Para ir despuntando nuestro recorrido, y como un estímulo para nuestros señalamientos y aseveraciones, nos detendremos especialmente en ciertos textos del psicoanalista francés Christophe Dejours, quien en su obra "Trabajo y Desgaste mental. Una contribución a la psicopatología del trabajo" (1980) inauguraría un modo de visualizar el campo de la Psicología del trabajo con características bien específicas.

En Dejours se daría un entronque entre un cierto psicoanálisis inspirado sobretodo en las teorizaciones de Melanie Klein y la experiencia del trabajo (psíquico) en el medio laboral. Este sería un punto importante y podría acercarnos también a comprender por que existe un fuerte desconocimiento del legado de Dejours en campos tanto clínicos y organizacionales como teóricos. El gesto es también el que recorre este artículo: dar pie a una apertura hacia otros alcances de la investigación psicoanalítica y psicosomática y de la investigación en Psicología laboral, buscando contribuir a replantear ciertas cuestiones que sólo podrían ser abordadas si existe una disposición hacia este diálogo.

Para dilucidar nuestra hipótesis de un vínculo entre el psiquismo y la institución del trabajo, recurriremos a remarcar los lugares en los que dicha ligazón se establece. Ciertas patologías que en el texto se hacen objeto de atención (las afecciones psicosomáticas, la ansiedad y la insatisfacción laboral), nos permiten hallar un compromiso entre el aparato psíquico del individuo y las situaciones en las que se define su trabajo de sustento. Allí es donde ciertos énfasis psicoanalíticos permiten dar una luz sobre el sufrimiento psíquico que un individuo sostiene en su puesto de trabajo.

\section{La institución del trabajo y el vínculo subjetivo.}

El hallazgo que Dejours pone sobre el tapete es un intento de mirar a través de un tratamiento psicoanalítico las problemáticas intersubjetivas que se ponen en juego en el trabajo, más precisamente, en la tarea específica que se desempeña en el día a día. Este hallazgo se ofrece, y será bueno sostenerlo de antemano, a precio de una reducción fundamental. La operación a discutir es una reducción en la cual la institución del trabajo es reducida a una situación de confrontación entre un medio social y lo psíquico. Hay dos registros, la institución y el psiquismo, que en su acercamiento se hacen temblar entre sí, puesto que "en el enfrentamiento del hombre con su tarea, pone en peligro su vida mental." (Dejours, 1992a, pág.9) Bajo este eje de análisis, la psicopatología laboral se vuelve punto importante de consideración al establecer, a partir del hallazgo del sufrimiento en los individuos, una continuidad entre este sufrimiento, como ligazón del orden psíquico-somático que sustenta la subjetividad in- 
dividual, y las condiciones que se establecen para llevar a cabo una tarea circunscrita en el medio laboral.

Habría, de este modo, una "continuidad" de carácter bien particular. Este vínculo se estaría mostrando como pasaje entre lo individual y lo social, allí donde la distancia de ambos parece generar un fenómeno nuevo. En este marco, y desde nuestra particular lectura, consideraremos a la tarea laboral como un caso excepcional de la introducción de la norma en el devenir subjetivo, una serie de normas asociadas entre sí, con vistas a mantener una actividad vital de sostén psíquico y socioeconómico. Entonces, la situación laboral como lugar excepcional para pesquisar el trabajo propio del psiquismo en el entorno social que es aquel que le da todo su sentido y su expresión.

La operación que nos parece interesante de rescatar es el intento de localizar al "síntoma" de este choque en un lugar descentrado y no individualizable por entero, enfatizando la relación producida bajo un determinado entorno laboral. Este es también el problema, en tanto se presenta como un ejemplo de lo institucional, pero reduciéndolo al delimitarlo únicamente al ejemplo presentado en la relación laboral. Esta determinación de un entorno que por excelencia se articula según sus funciones y operaciones precisas hace parecer que de lo que se habla es de una organización social específica y no directamente del peso que ella estaría imprimiendo sobre toda actividad, peso que es propiamente la institución y que actúa sobre otras dimensiones además de la organización social. Sin embargo, es la inserción de la normativa en lo subjetivo -una "normativización del psiquismo"- aquello que da cuenta de ciertos modos de atravesamiento de la institución en el psiquismo y de la configuración de éste en virtud de la institución del trabajo, esto es, la configuración que recibe el psiquismo en su contacto con un cierto orden establecido según las tareas precisas que un sujeto debe desempeñar en un puesto de trabajo. Es aquí mismo donde se abriría una puerta de entrada para definir una psicología en términos liminares y no puramente centrada en el individuo.

En el medio de la tarea que debe desempeñar el trabajador en su puesto laboral, el énfasis de Dejours se establece sobre el encuentro entre el comportamiento y la ejecución de la tarea, considerando la eventual flexibilidad o falta de ella en esta relación. El sufrimiento se sitúa en dichos movimientos que constituyen la tarea, la ligazón hombre-tarea. El sufrimiento es una ausencia, pero es ausencia de comportamiento libre (Ibíd., p.28), la cual en su permanencia y en su continuidad -de la tarea que se lleva a cabo- va a mantener el sufrimiento en este medio. El sufrimiento, bajo esta mirada, va a ser designado según un empobrecimiento en el compromiso de lo psíquico con la institución del trabajo, allí donde ella no se flexibiliza frente a las capacidades de lo psíquico, a la creatividad que el psiquismo imprime al trabajo.

Para hacer patente lo dicho hasta aquí, Dejours va a localizar su investigación en un sector específico, el de los trabajadores industriales, para mostrarlo como lugar en donde el sufrimiento aparecería más marcado y visible. En este grupo de trabajadores la enfermedad mantiene una relación unívoca a la interrupción del trabajo: el cuerpo se hace visible sólo cuando es posible que éste hable desde un dolor. (Ibíd., p.36) Es en los trabajos más pesados -la construcción y las industrias químicas son los ejemplos a los que el autor nos remite- donde se requiere de un paso intermedio, un punto de mediación de este dolor. Para ello, se constituye una suerte de ideología defensiva, la cual "consiste en mantener alejado el riesgo de un agotamiento del cuerpo que lo aleje del trabajo" (Ibíd., p.38) Lo central en dicho mecanismo es que no se constituye como un mecanismo de defensa individual -a la manera de los 
descritos por Anna Freud ${ }^{2}$, por ejemplo-, sino como una verdadera ideología defensiva en tanto dimensión especifica que se instala como resistencia colectiva frente a una cierta situación que puede llegar a desencadenarse en el trabajo, resistencia colectiva frente a lo que puede llegar a ser: resistencia a la amenaza de desbordamiento del psiquismo frente a una situación incontenible y amenazante. Esta ideología defensiva presenta dos ejes fundamentales que es preciso resaltar. Por un lado, se rescata el tema de la defensa, la cual actúa como un punto intermedio entre el sufrimiento efectivo y las condiciones laborales de su producción. Por otra parte, se rescata a lo colectivo como aquel medio en el cual se constituye esta estrategia defensiva, lo cual lleva a considerar que el lugar que ella circunscribe no posee un dueño, a la manera en que se podría ser "dueño" de una cierta sintomatología clínica. No, Dejours da cuenta que si bien esta sintomatología aparece en cada individuo, esta responde a un lugar común. Tal como la organización científica del trabajo propuesta por Taylor, "individualiza a los hombres frente al sufrimiento" (Ibíd., p.44), aquí el mecanismo pesquisado remite directamente a las relaciones propias de una estructuración particular del trabajo la cual es, por entero, colectiva.

Las distinciones entre trabajador y trabajo, entre psiquismo e institución, se pondrían en suspenso, mostrando algo más primordial a toda oposición: la relación laboral hace exhibir el enfrentamiento de dos tipos de registros: el psiquismo y la institución. El enfrentamiento a la tarea hace que el sujeto se movilice en dos planos. Por un lado, una tarea se impone a su psiquismo, apareciendo como una imposición exterior a él. Por otra parte, resulta ser que las tareas que constituyen la actividad laboral se ven vinculadas, separándose, al trabajo psíquico que marca sus propias pautas. Es allí donde surgiría el sufrimiento que el sujeto experimenta.
Es allí mismo donde el psicoanálisis podría llegar a proponer los instrumentos conceptuales para enriquecer lo que en la actividad laboral ocurre, haciendo visible, dando otra visibilidad a la compleja articulación de relaciones entre los aspectos psíquicos que impulsan al trabajador y las situaciones en que éste se ve comprometido. Detengámonos sobre esto más de cerca.

\section{Insatisfacción y ansiedad. La difícil vinculación entre psique-soma e institución.}

El modo de lectura del sufrimiento que se produce en el trabajo y que va a ser ligado directamente a la relación entre condiciones de producción subjetivas y sus espacios laborales correspondientes (de distinto tipo: psíquico, lugar físico, tipo de tarea a ejecutar, etc.) opera por el develamiento de ciertos síntomas claves a través de los cuales podrán aparecer dos modos de sufrimiento fundamentales que competen al psiquismo de los involucrados: la insatisfacción y la ansiedad.(Ibíd., p.55 y ss.) Discutiremos como eje central ambas formas del sufrimiento en el mundo del trabajo y, en su transversalidad, buscaremos esbozar ciertas determinaciones teóricas para una puntualización de carácter más general sobre el sufrimiento psíquico en la relación con la institución del trabajo, enfatizando asimismo cómo se logra ver allí la relación entre el psiquismo, el cuerpo y la institución.

\section{a) La Insatisfacción en el enfrentamien- to del hombre a la tarea}

La insatisfacción frente a la tarea que el trabajador desempeña se constituye como un polo de origen del sufrimiento. Más que predecir, la insatisfacción experimentada muestra una imposibilidad de convergencia entre los objetivos de la tarea y sus resultados. Así, entra en pugna el nivel de calificación y de formación requerido en un puesto de trabajo, frente a las aspiraciones particulares de los trabajadores. Precisamente, "el sufrimiento 
comienza cuando la evolución de esta relación está bloqueada" (Ibíd., p.57) El bloqueo genera una distancia entre el requerimiento del espacio laboral y el requerimiento efectuado por el psiquismo. En ese intermedio, el sufrimiento anuda ambos espacios de un modo violento; el psiquismo no se adapta a la normativa impuesta por una exterioridad a él, no se adapta a sus deberes. Si la especificidad de lo psíquico es presentarse también como "ateniéndose a una cierta norma", como dirigido por un orden propio, asistimos aquí a un choque entre dos dimensiones normativas. Pero, en este choque, el trabajo del espacio psíquico es absorbido por la figura las funciones- del trabajo que debe ser desempeñado en un puesto de trabajo: Las normas productivas son también normas mentales. (Ibíd., p.145)

En este "encuentro" ocurre una singular figurabilidad del mismo. Lo que sobra, lo excedente, es la tarea impuesta que se desempeña en un puesto de trabajo y que muestra al psiquismo ordenado desde ciertos patrones exteriores a él. Este excedente permanece bajo la no-simbolización. En este sentido, la incapacidad de simbolizar lo excesivo de esta tarea para el psiquismo, su incapacidad de adecuación a la tarea, estaría mostrando que no hay una apropiación del psiquismo sobre la tarea en la cual se debe desenvolver: el significado del trabajo es aquello insimbolizable, sometido a las puertas exteriores de lo psíquico y generando un sufrimiento fundamental por la discordancia entre la tarea y su incapacidad de integrarla bajo una significación. En este punto se aloja un sufrimiento, como cruce entre el trabajo del psiquismo y las condiciones del puesto de trabajo.

Este sufrimiento que se deja sentir en la dimensión psíquica, no actúa como oposición frente a un posible placer absoluto y total en la tarea que se despeña. Este sufrimiento estaría dado por la incapacidad de cambiar la tarea (Ibíd., p.60) que se desempeña, tarea que se busca flexibilizar pero que nunca es totalmente adecuada para las exigencias del psiquismo: Hay algo que resiste la articulación. Pareciera ser que lo psíquico actuaría en gran medida como un flujo que no se deja aprehender por otra normativa fuera de la suya. El error sería creer que esta normativa es algo natural: se puede intuir que es precisamente la presencia de estas imposiciones y ordenamientos aquello que da una cierta "identidad" al psiquismo, imposiciones que no son sino el mismo movimiento del principio de realidad, descrito por el psicoanálisis.

Se trataría de mostrar esta movilización de lo psíquico que hace imposible aprehenderlo más que por sus propios y siempre desmarcatorios ritmos. Lo que reclama sería la sujeción, no la actividad que todo trabajo supone. Un respeto por el trabajo psíquico con otro tiempo que el "otro" trabajo. De este modo, el sufrimiento mismo sería índice o indicador de la división del trabajo, del rango de distanciamiento entre las tareas que componen a un sector de la división y aquello que el psiquismo tolera. Habría una relación indisoluble entre el sufrimiento, el tipo de división del trabajo y el contenido de la tarea que se desempeña: si ésta es susceptible de ser significado por el psiquismo del sujeto, y significativo afectivamente. $\mathrm{Si}$ el sufrimiento deviene a través de la incapacidad de modificación de la tarea en el trabajo, lo fundamental "no es tanto la importancia de exigencias mentales o psíquicas del trabajo las que hacen aparecer el sufrimiento (por más que el factor aparezca como evidentemente importante) sino más bien la imposibilidad de toda evolución para aliviarlo."(Ibíd., p.60)

Sería la incapacidad de control de las exigencias efectuadas por el psiquismo aquello que se traspondría como sufrimiento en la actividad que se desempeña. Sin embargo, no se trataría de reducir al psiquismo a aquello que a éste le sea soportable dado que parecieses haber aquí algo irreductible. Si la inscripción innegable y radical de un conflicto es condición de su posibilidad de ser 
psíquicamente integrable (Duez, 202 y s.), habría algo en el sufrimiento experimentado por el hombre al enfrentarse a su tarea que nos haría suponer una imposibilidad de asunción de un conflicto. Aquí se trataría de la imposibilidad de reconocimiento de una institución en el mismo psiquismo; institución y ritmo de lo psíquico irreductible al trabajo que le es ajeno.

Es el choque entre los dos registros mencionados -un trabajo del psiquismo y un trabajo sometido al régimen del salario- aquello que define el sufrimiento. Allí donde éste aparece, es porque yace la alteración entre la necesidad de gratificación y el cumplimiento de dicha necesidad. En esta alteración, el sufrimiento no se somete meramente a lo psíquico. Su expresión también puede aparecer corporalmente, eso sí vía la mediación del psiquismo. Lo social representado por la vivencia que impone al psiquismo va a aparecer en el cuerpo orgánico (Dejours, 1992a, p.71). El trabajo se acopla al cuerpo, constituyéndolo como tal.

El contenido de la tarea, su capacidad de ser significado o no por el sujeto que desempeña la tarea, puede generar una insatisfacción debido al carácter de la tarea, el cual es siempre inadecuado a lo psíquico que más que por necesidades actuaría movido por el deseo, o como dirían Deleuze y Guattari por un "deseo de deseo", que al no cumplirse puede llegar a ser aprehendido como insatisfacción que se moviliza en el espacio psíquico. Sin embargo, este espacio psíquico se excede a sí mismo, al mostrarse indisociablemente ligado al cuerpo en tanto el sufrimiento de este tipo, "resultante de una frustración al nivel del contenido significativo de la tarea puede igualmente conducir a afecciones somáticas." (Ibíd., p.72) Pero no se trata de una cuestión puramente unidireccional. No solo lo psíquico incide sobre lo somático. En su desborde, toda condición, y toda relación posible en el trabajo, se constituirá también en "una carga de trabajo psíquico" (Ibíd., p.72), configurando al psiquismo en una difícil relación con la situación laboral.

La afección que se expresa somáticamente y que nos conduce, en su origen, a ciertas condiciones bajo las cuales el aparto psíquico se vería afectado, se presenta como un lugar privilegiado para establecer la articulación entre los instrumentos conceptuales ofrecidos por el psicoanálisis y las situaciones a las que se enfrenta la Psicopatología del trabajo en su quehacer cotidiano. Es así como ciertas enfermedades nerviosas, situaciones extremas de estrés laboral o incluso el llamado Burn-out pueden llegar a ser explicados según el esquema propuesto por Dejours.

Lo psicosomático sería, pues, lugar esencial para pesquisar algo así como el "desentendimiento" con respecto al sufrimiento en el plano de lo psíquico. En otro lugar, Dejours señala que las relaciones entre el psiquismo y el cuerpo conciernen "esencialmente a los efectos patogenosos de ciertos acontecimientos psíquicos sobre la salud del cuerpo" (Dejours, 1992b, p.104) La primacía sería, en este sentido, del ámbito del psiquismo. Lo psíquico podría poseer una plasticidad tal que lograría esta desestabilización sobre lo somático. De algún modo, en este proceso, este desentendimiento de cierto sufrimiento psíquico aparecería, por economía, en el cuerpo. El sufrimiento se inscribiría en el cuerpo, sosteniendo al sujeto y evitando su muerte física. En el trabajo la aparición de mareos, migrañas, problemas estomacales, y otras formas, estarían mostrando la resistencia del cuerpo frente a esta dificultad de conciliación de ambos registros mencionados. Un exceso de simbolización de ese malestar difuso, que al parecer no se puede designar ni desconocer.

En la afección psicosomática se cristaliza una interrogación por los límites del vínculo psíquico al bordear toda institución, todo ordenamiento para el psiquismo de un sujeto -el establecimiento de la norma, no im- 
porta cual sea, sobre él. Por donde se aborde, lo psicosomático es exposición a los límites que se le imponen: indisociabilidad entre el cuerpo y la psique como dos órdenes con leyes propias, e interrupción de la institución del trabajo en la intimidad del vínculo psique-soma. El cuerpo se ve saturado al no dejarse "equilibrar" con lo psíquico; pudiendo tratarse de una incapacidad de presentar algo en el nivel de lo psíquico parece hacerse patente en otro nivel, en este caso el cuerpo (Abraham, 1969) o bien, por un sentido que se hizo desmesurado para el psiquismo, tal como la presión que se puede ejercer en una situación laboral que genera malestar y que se ve prolongada.

La aporía de la afección psicosomática nos pone frente a aquello que nos hace imposible decidir por la primacía de uno sobre otro, de lo psíquico por sobre lo somático. No se trata entonces de una pérdida de la homeostasis, sino más bien en un conflicto que es indisoluble y que en su máxima expresión constituye lo psicosomático, como un desconocimiento del cuerpo de un sujeto de parte de su propia capacidad psíquica. Valga recordar aquello que Jacques Lacan viene a precisar: "La psicosomática es algo que no es un significante, pero que, sin embargo, sólo es concebible en la medida que la inducción significante al nivel del sujeto ha transcurrido de un modo que no pone en juego la afánisis del sujeto." (Seminario XI) Lo psicosomático se hace representable solo a través del significante-síntoma, pese a que este medio no le sea el más adecuado. Esto mostraría de manera excepcional el hallazgo del psicoanálisis: un clivaje subjetivo que instala en el seno de la subjetividad al desconocimiento y el desarraigo. El análisis de la actividad laboral -por ejemplo, de las frustraciones experimentadas en la tarea que se desempeña en el puesto de trabajo-, propone ser pensado como una difícil situación en la cual se compromete lo mas profundo del sujeto en su encuentro con las reglas que allí le son impuestas.

\section{b) La ansiedad como anticipo al riesgo}

En el polo de la ansiedad, como otra forma de aparición del sufrimiento en relación con las condiciones de trabajo, se podría decir que, a diferencia de la insatisfacción, la ansiedad se genera como una suerte de $a$ priori frente al desempeño efectivo de la tarea asignada al psiquismo. La ansiedad es sencillamente la incapacidad, colectiva, de controlar el riesgo posible de toda situación laboral (Dejours, 1992a, p.77). En la medida en que nadie debería temer en su puesto de trabajo, en ello mismo reside el control por parte de una ideología defensiva que impide tocar cualquier tema relacionado con la inminencia de esta posibilidad, haciendo impensable llevar a cabo un trabajo sobre dicha ansiedad, la cual es vivida como efecto irreparable e irreversible de las condiciones de trabajo específicas. En la medida en que se transforma en una presencia constante de lo propiamente irrepresentable -el despido, el accidente o, incluso, la muerte- se constituye como un coeficiente que se multiplica a sí mismo. La ansiedad actúa sobre ese fondo vacío, el cual "aumenta también el costo mental o psíquico del trabajo." (Ibíd., p.79)

La organización del trabajo logra penetrar toda la vida y allí la ansiedad reina más que por la realización de sus promesas, por su constancia. La ansiedad aparece como forma psíquica de sufrimiento anticipado frente a aquello que se avecina, aquello que puede ser posible de forma permanente. En una consideración económica, si aquello no aparece en el cuerpo, el riesgo y su posibilidad se vive como ansiedad. La ansiedad es amenaza constante de lo por-venir, surtiendo sus efectos antes de cualquier futuro. Parece ser que mientras en la insatisfacción existe un dejo de fracaso en la realización de la actividad que no puede ser absorbida del todo por lo psíquico, en la ansiedad se estaría poniendo en juego la probabilidad inminente de que la tarea que se desempeña termine por eliminar la actividad del psiquismo, sea en una anulación metafórica como en los tra- 
bajos repetitivos e inflexibles, sea en un sentido literal con el anticipo de la propia muerte por alguna dolencia o enfermedad producto de la tarea.

\section{c) Defensa e institución}

Frente a ambas consideraciones sobre el sufrimiento en el espacio laboral -la insatisfacción y la ansiedad-, se constituye una ideología defensiva que, más que buscar lograr una superación o un tratamiento de dichas formas y sus orígenes, se explota más allá de sí misma (Ibíd., p.124 y s.). Se transforma en un plus: Este sufrimiento cohesiona a través de sus defensas colectivas, pero asimismo, genera un excedente al usufructuar de la imposibilidad de su resolución efectiva. En el uso de las defensas frente al sufrimiento, se desarrolla una posibilidad de unión de los trabajadores (Ibíd., p.183), lo cual daría cuenta de una función más positiva sobre este sufrimiento.

La institución misma, en este caso aquellas tareas que el trabajador desempeña en su puesto de trabajo, con todos los valores y aspiraciones que se ponen allí en juego, actúa como una defensa frente a algo más primordial (Jaques, 1970). La institución es una defensa contra la violenta irrupción del psiquismo que amenaza con desbordarse sin norma alguna sobre el campo social. Al ponerse en común las fantasías que inspiran la sensación de posible ruptura, se puede decir que la defensa es por esencia colectiva. El sufrimiento en el trabajo es una situación límite, puesto que, en tanto medio de subsistencia no es posible prescindir de él. Ello afirma la permanencia del sufrimiento en él. Frente a esto, se establece un modo de hacer colectivo este sufrimiento que compromete, de hacerlo sostenible en el espacio común propio del trabajo. Tal vez aquí se podría vislumbrar al sufrimiento como una constante, en cierto grado, de toda la existencia social. En esta medida, el sufrimiento no sólo sería un déficit.
"Incluso cuando el sufrimiento es intenso, es bastante bien controlado por las estrategias defensivas para impedir que pase a adoptar forma de patología."(Dejours, 1992a, p.144) Esto deja en claro que el sufrimiento mismo, por medio de sus defensas que aparecen colindantes a él, genera la ganancia de un cierta estabilidad a no pasar hacia la locura o la muerte. Así, se termina con establecer la ideología defensiva que, en muchos casos, juega en contra de la superación de este sufrimiento. Muestra la imposibilidad total de la satisfacción y frente a esa imposibilidad instala la simbolización extrema de lo psicosomático o la ansiedad flotante y permanente, desconociéndose en ambas el origen y la mantención del sufrimiento. En la medida en que las defensas logran anestesiar el sufrimiento, allí las defensas se pueden llegar a transformar en radical resistencia al cambio (Ibíd., p.184).

A esta altura, en el momento en que la investigación llevada a cabo por Dejours sobre la relación entre el psiquismo y la institución del trabajo logra replantear las relaciones entre el psiquismo y el orden de lo somático, se puede llegar a considerar la intuición de un atravesamiento de la institución en el psiquismo. Lo psicosomático y la ansiedad como manifestaciones ligadas al sufrimiento en el trabajo, al choque entre el psiquismo y el entorno laboral, no son nunca manifestaciones individuales, por mucho que sean identificables solo en la particularidad de cada individuo. La institución anuda el trabajo al psiquismo, y en ello involucra muy de cerca al cuerpo. No es posible disociar, salvo bajo una operación de análisis, el par psique-soma: como ya se aludió, lo social se inscribe en el cuerpo.

En este atravesamiento, el sufrimiento se transforma en puente de articulación, en un nudo de cohesión frente a la situación extrema del sometimiento del psiquismo a la institución del trabajo, sometimiento ineludible en cierto sentido. Lo sufriente es la inadap- 
tación cabal del "mundo" psíquico, el cual está hecho en ciertos aspectos, "a imagen y semejanza" del trabajo al cual es sometido. El psiquismo sufre por la imposibilidad de adecuación. El sufrimiento en este atravesamiento es un permanente "estado de equilibrio inestable" (Ibíd., p.182), en el cual reside alguna posibilidad de evitar la destrucción total sobre la base de la formulación y el mantenimiento de defensas que sostengan, en el sufrimiento, al propio psiquismo.

\section{Consideraciones finales. El aporte de Dejours a la comprensión de la actividad laboral.}

En la Psicopatología laboral, el movimiento que durante el enfrentamiento del sujeto a su tarea en un determinado puesto de trabajo desprende algún tipo de malestar se puede ofrecer acaso como una instancia teórica y empírica, por cierto- excepcional para lograr capturar una especial mirada sobre el vínculo entre la institución y el psiquismo. Antes que nada, habría que desechar una cierta limitación del problema al campo específico de su emergencia, ateniéndonos a los peligros que esto conllevaría de antemano.

Tal vez el gesto de la mirada que Dejours nos propone deba ser leído como unos apuntes que intentan sugerir distintas herramientas de análisis que, en su puesta en discusión, busquen extender los problemas cotidianos no sólo de la Psicología del trabajo, sino también de los propios trabajadores, a ser considerados como cuestiones que competen a todo el ámbito individual y social.

Es así como la iniciativa de Dejours, que trabaja ante todo un enfrentamiento del psicólogo a la experiencia vivida por los trabajadores en sus lugares de trabajo, viene a instalarse como una alternativa legítima frente a las categorías más tradicionales de la Psicología y la Psicopatología del Trabajo. Por ejemplo, allí donde se hace visible el estrés laboral, Dejours propone una articulación entre la ansiedad y la insatisfacción que el trabajador experimenta al desempeñar su tarea.

Las dilucidaciones de Dejours comprometen una vía de investigación de sumo interés tanto para la Psicopatología del Trabajo como para, en su posibilidad de encuentro, el psicoanálisis.

Lo que el trabajo de Dejours viene a cuestionar es el cierre que existe, en el trabajo mismo del psicólogo al crearse áreas separadas tajantemente. No se trata de aplicar categorías del psicoanálisis para hacer clínica psicoanalítica al interior de una organización. Mas bien, lo que se propone Dejours es venir a enriquecer y a ampliar, por medio de estas categorías, la manera como se abordan los fenómenos psíquicos en las organizaciones. Allí puede ser entendido que la experiencia sobre la que Dejours fundamenta su análisis no sea distinta de la experiencia a la que se enfrenta cualquier psicólogo preocupado de los fenómenos laborales y organizacionales: se trataría sobretodo de proponer una integración y una amplitud frente a una serie de hechos complejos como son los que se ponen de manifiesto al examinar los aspectos psíquicos de la actividad laboral.

Las consideraciones efectuadas con anterioridad apuntaban a dar la posibilidad a un diálogo que, tal vez sin haberse perfilado ni enfatizado en el modo en que lo hemos hecho, haga tratables distintas cuestiones sobre el vínculo entre lo psíquico y la situación laboral. Si la actividad humana es siempre enfrentamiento a ciertas tareas en las cuales se compromete el psiquismo, la situación laboral debe ser no sólo un ejemplo de ello, sino también una via regia que haría surgir aspectos que les son propios sólo a ella. Para ello baste con recordar las nuevas líneas de indagación que estarían sugiriendo una consideración de lo psicosomático, de la ansiedad o de la insatisfacción, como experien- 
cias producidas en y por el entorno laboral, obligándonos a echar una mirada sobre las complejidades del vínculo entre el trabajo y el psiquismo.

Al verse alterada en alguno de sus polos, la ligazón psique-soma atestigua por la irrupción de la institución del trabajo, la cual otorga su carácter especifico al sufrimiento. Lo expuesto por la Psicología del Trabajo de Dejours supone no sólo proyecciones para el trabajo empírico de intervención en la relación hombre-tarea, sino también una apertura para la discusión conceptual en torno a categorías no reductibles ni equiparables entre sí: la relación entre el psiquismo y la institución, entre el individuo y la sociedad, y entre el psiquismo y el cuerpo.

Se cumple entonces, en el recorrido que intentamos transitar desde nuestra lectura de Christophe Dejours, que este "diálogo" entre el psicoanálisis y la Psicología del Trabajo, con todos sus posibles asertos y paradojas, puede hacer visibles cuestiones no consideradas hasta el momento por estas maneras de concebir lo psíquico si se las considera aisladamente. Este encuentro es un lugar excepcional para pensar la relación existente entre el sufrimiento psíquico y la actividad laboral, y, por supuesto, para concebir los modos de enfrentamiento de las comunes inestabilidades que el sujeto experimenta en su puesto de trabajo.

\section{Referencias}

Abraham, N. (1969). "Reflexiones fenomenológicas sobre las implicaciones estructurales y genéticas del psicoanálisis", en Bloch, Piaget, y otros (1969). Las Nociones de estructura y génesis. Buenos Aires: Proteo.
Dejours, C. (1992A). Trabajo y desgaste mental. Una contribución a la psicopatología del trabajo. Buenos Aires: Credal-humanitas. (orig: 1980)

Dejours, C. (1992в). Investigaciones psicoanaliticas sobre el cuerpo. Supresión y subversión en psicosomática. México: Siglo XXI Editores. (orig: 1989)

Dejours, C. (1988). Plaisir et souffrance dans le travail. Paris: Ed. de l'AOCIP.

DuEz, B. "Psicopatología de lo imaginario y tratamiento de la figurabilidad. Elementos para una práctica psicoanalítica en institución", en Kaës, R. y otros (1998). Sufrimiento y psicopatología de los vínculos institucionales. Elementos de la práctica psicoanalítica en institución. Buenos Aires: Paidós. (orig: 1996)

JAQUES, E. (1970). “Los sistemas sociales como defensa contra las ansiedades persecutoria y depresiva. Una contribución al estudio psicoanalítico de proceso sociales", en Menzies, I. y E. Jaques, Los sistemas sociales como defensa contra la ansiedad. Buenos Aires: Paidós. Buenos Aires: Editorial Paidós. (orig: 1955)

LACAN, J. (1995). El Seminario. Libro 2: El Yo en la Teoría de Freud y en la Técnica Psicoanalítica. Buenos Aires: Paidós. (orig: 1978)

LACAN, J. El Seminario. Libro XI. Los cuatro conceptos fundamentales del psicoanálisis. Buenos Aires: Paidós.

2 Véase a este respecto y para mostrar mas claramente la noción de mecanismo de defensa frente a lo por Dejours propuesto: Freud, Anna (1961). El Yo y los mecanismos de defensa. Buenos Aires: Paidós 